\title{
Psychothérapies des patients atteints de cancer : le vrai et le faux
}

\section{Cancer patients and psychotherapy: distinguishing truth from falsehood}

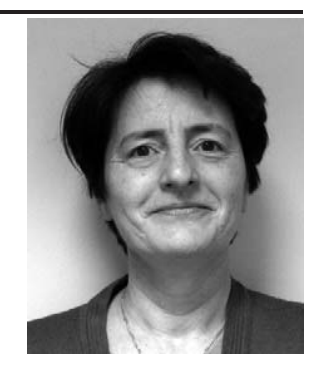

\author{
N. Pélicier \\ (C) Springer-Verlag France 2010
}

Le récent décret d'application de la loi 2004, relative à l'usage du titre de psychothérapeute, a suscité des réactions hostiles parmi de nombreuses associations de psychologues cliniciens et de psychothérapeutes (psychiatres et psychologues). La Société française de psycho-oncologie (SFPO) s'est associée à la pétition de nos collègues psychologues cliniciens pour la révision du décret.

Pourquoi confondre la durée de formation (demande de complément en heures de psychopathologie) et son contenu (nécessairement révisable, renouvelable) ?

Pourquoi décider que le type de cursus (master de psychologie clinique et de psychopathologie), validé et utilisé jusque-là, deviendrait en partie caduc ?

Le foisonnement de "praticiens » en psychothérapies, sans contrôle, la réalité de pratiques douteuses a motivé le législateur, comme dans d'autres professions visant le " soin », à rechercher à établir des critères de formation pour prétendre au titre de psychothérapeute. Dans cette nécessaire et salutaire explicitation destinée au public et aux professionnels, des psychiatres et des psychologues se sont sentis gravement remis en question.

La volonté d'assainir des pratiques, qui, précisément n'appartiennent pas strictement au champ clinique qui est bien le nôtre en psycho-oncologie, vient ainsi, bien maladroitement et paradoxalement, jeter le trouble sur ce qui fonctionnait bien jusque-là avec l'aval des universités et des filières officielles de formation en soins psychiques. Pour le public d'usagers, il sera difficile de s'y retrouver.

Le champ de la cancérologie est régulièrement traversé par des interventions peu structurées, qui se veulent des solutions thérapeutiques au «malaise psychique » des patients. Groupes de paroles non menés par des psys, lieu d'échanges sur le vécu sans cadre professionnel à même d'accueillir les réactions liées au vécu de groupe, constituent peu à peu des

\section{N. Pélicier $(\bowtie)$}

Hôpital européen Georges-Pompidou (HEGP),

20, rue Leblanc, F-75908 Paris cedex 15, France

e-mail : nicole.pelicier@egp.aphp.fr propositions d'aides peu définies, sans qu'aucun critère ne soit demandé pour les proposer aux patients ou à leurs proches. Les injonctions sont multiples en situation de vulnérabilité dans la maladie grave, pour imposer aux malades d'assumer la responsabilité de se tirer d'affaire psychiquement.

Nous avons vu «fleurir » et se développer depuis 30 ans en France et ailleurs, de véritables lobbies de l'aide psychique dont les buts ne cessent de nous interpeler, n'excluant souvent ni le profit, ni la volonté d'emprise.

Nous sommes donc bien d'accord pour une vigilance que soutiennent les Miviludes (mission interministérielle de vigilance et de lutte contre les dérives sectaires), il convient alors de redire à nos patients et à nos institutions que les psychothérapies ne s'inventent pas au détour d'une souffrance (anciens malades proposant d'animer des groupes de patients, de professionnels de santé confondant leur propre besoin dans un parcours difficile et l'aide destinée aux patients...).

De façon plus défensive, mais aussi de notre fait ou de notre faute, nos hôpitaux, nos services se chargent de recruter et d'absoudre des intervenants peu formés, nonpsychothérapeutes, pour lesquels leurs histoires émouvantes, leurs bonnes intentions..., tiennent lieu de formation.

Notre société savante (SFPO) cherche depuis longtemps à promouvoir la qualité des soins psychiques proposés aux patients sous forme de psychothérapies. Même si nous ne représentons pas la seule source d'aide psychologique reçue par les patients, le soin psychique est bien notre affaire.

Le contenu des formations doit rester, pour cela, en lien étroit avec le champ des demandes psychothérapeutiques de plus en plus manifestes en médecine. Nous souhaitons que l'on reconnaisse qu'un(e) psychologue clinicien(ne) ou un(e) psychiatre dont les diplômes sont reconnus officiellement, ne sont pas à mettre dans le même volet de compétence que les intervenants dont les croyances ou les succès médiatiques tiennent lieu de formation.

Nous sommes donc assez "interloqués », au-delà du débat autour de la pétition de nos collègues psychologues 
cliniciens, à laquelle la SFPO s'est associée, de l'ignorance des pratiques régulières et validées de psychothérapies dans le champ de la psycho-oncologie au profit du vaste champ de «l'aide psychique pour guérir » (sites Web) en cancérologie, dont l'évaluation semble particulièrement absente. C'est cette tolérance du flou, cette déspécification et ce manque de professionnalisme en matière de soins psychiques vis-à-vis duquel nous voulons être plus que jamais vigilants.

Ce numéro de la revue de la Psycho-Oncologie a l'ambition de présenter l'épanouissement et le regain nécessaire des formes reconnues et actualisées de psychothérapies en cancérologie. La restitution de nos connaissances et des meilleures pratiques suscitera, nous l'espérons, plus de curiosité et de respect pour nos collègues psychothérapeutes en psycho-oncologie.

Conscients qu'un décret ne résout pas tout, nous continuons à la SFPO, à proposer des formations spécifiques qui ne garantissent pas seulement en nombre d'heures accomplies la qualité de formations mais plutôt en restituant une expertise et une expérience clinique validées.

Merci de ne plus confondre les professionnels et les autres, le vrai et le faux. 